\title{
Data-based control, optimization, modeling and applications
}

\author{
Dongbin Zhao $\cdot$ Yi Shen $\cdot$ Zhanshan Wang $\cdot$ \\ Xiaolin Hu
}

Published online: 4 January 2013

(C) Springer-Verlag London 2013

We are honored to organize this special issue of Neural Computation and Applications, based on contributions to the Ninth International Symposium on Neural Networks (ISNN 2012) held on July 11-14, 2012, in Shenyang, China. Evaluated by the contributions and the recommendation of ISNN 2012 organizers, 22 papers were selected for the further review process with their extended form. Each paper has been reviewed thoroughly by three independent experts in related research area. We are very grateful for the hard work of the reviewers, which help to facilitate the outcome of this special issue with greatly improved quality of the contributions. Finally, 20 papers are accepted, to be introduced as follows.

\footnotetext{
D. Zhao $(\bowtie)$

State Key Laboratory of Management and Control for Complex Systems, Institute of Automation, Chinese Academy of Sciences, Beijing 100190, China

e-mail: Dongbin.zhao@ia.ac.cn

\section{Y. Shen}

Key Laboratory of Ministry of Education for Image Processing and Intelligent Control, Department of Control Science and Engineering, Huazhong University of Science and Technology, Wuhan 430074, China

e-mail: yishen64@163.com

\section{Z. Wang}

School of Information Science and Engineering, Northeastern University, Shenyang 110004, China e-mail: zhanshan_wang@163.com

X. Hu

State Key Laboratory of Intelligent Technology and Systems, Tsinghua National Laboratory for Information Science and Technology, Department of Computer Science and Technology, Tsinghua University, Beijing 100084, China

e-mail: xlhu@tsinghua.edu.cn
}

In brief, the contributions of this special issue can be roughly classified into three major categories: data-based control, data-based optimization, and data-based modeling. In each category, not only the theoretical foundation is analyzed, but also some prospective applications are demonstrated. In this introduction, we like to borrow some ideas from the contributions, such as hierarchical, and cluster as the subsidiary category idea.

The first category consisting of 6 papers is devoted to the data-based control and applications area, by which an optimal or near-optimal control can be derived from the input and output data. The first 4 papers focus on the hot research direction, adaptive dynamic programming and reinforcement learning (ADPRL), and the remaining 2 papers relate to iterative learning control and optimal control.

Derong Liu et al. develop an online algorithm based on policy iteration for continuous-time optimal control with infinite horizon cost for nonlinear systems. In the proposed method, a discount value function is employed, which is considered to be a more general case for optimal control problems. Meanwhile, without knowledge of the internal system dynamics, the algorithm can converge uniformly online to the optimal solution of the modified HamiltonJacobi-Bellman equation. By means of two neural networks, the algorithm is able to find suitable approximations of both the optimal control and the optimal cost.

Dual iterative adaptive dynamic programming for a class of discrete-time nonlinear systems with time-delays is investigated by Qinglai Wei et al. Dual iterative ADP algorithm is introduced to obtain the optimal solutions of the optimal performance index function and control, where in each iteration, the performance index function and the system states are both updated. Convergence analysis is presented to prove the performance index function to reach 
the optimum by the proposed method, in which neural networks are used to approximate the performance index function and compute the optimal control policy, respectively.

Optimal control problem of an electric vehicle's charging schedule under electricity markets is investigated by Tian Lan and Junjie Hu et.al. Since the hourly electricity prices are different in the market, the charging costs for electric vehicles generally differ from each other in choosing the charging time interval. This problem is formulated into an optimal control problem, and then a dynamic programming approach is proposed to solve the optimal charging solution for each vehicle. With smart charging, electric vehicles are recharged during the lowest electricity price period, which is also the off peak hours. It naturally drops the possibility of grid overload during the peak load hours.

A novel hierarchical reinforcement learning approach for optimal path tracking of wheeled mobile robots is proposed by Lei Zuo and Xin Xu et al. In the proposed approach, a graph Laplacian-based hierarchical approximate policy iteration (GHAPI) algorithm is developed, in which the basis functions are constructed automatically using the graph Laplacian operator. In GHAPI, the state space of an MDP is divided into several sub-spaces and approximate policy iteration is carried out on each subspace. Then, a near-optimal path tracking control strategy can be obtained by GHAPI combined with proportionalderivative (PD) control. The performance of the proposed approach is evaluated by using a P3-AT wheeled mobile robot. It is demonstrated that the GHAPI-based PD control can obtain better near-optimal control policies than previous approaches.

Iterative learning control aims at enhancing the tracking performance of systems with repetitive tasks. During decades, many adaptive iterative learning control algorithms have been developed. However, the system convergence and stability are not resolved satisfactorily. In this paper, Xiong xiong $\mathrm{He}$ et al. present an adaptive iterative learning control algorithm based on boundary layer for trajectory tracking of uncertain robot system. Sliding mode variable structure control is used to improve the robustness to disturbance and perturbation, and boundary layer is used to eliminate the chattering of sliding mode control. In the iterative domain, the unknown parameters are tuned. The stability and convergence of the algorithm are analyzed by using a Lyapunov-like method.

Polymer composite materials have achieved widespread use in industry, including automobile, aviation, and construction. Their manufacture involves thermoset curing which is dominated by complex dynamics. Trial and error has been the only practical optimization method to improve process operation. Kazim-Junior Mohammed and Jie
Zhang present a study on the optimization control of a reactive polymer composite molding process using ant colony optimization and bootstrap aggregated neural networks. Neural networks are deployed to model reactive polymer composite molding processes while ant colony optimization is used to find the optimal curing temperature profile. It is also found that incorporating model prediction confidence bound in the optimization can improve the reliability of the calculated optimal control policy.

Another 7 papers are listed in the second category, databased optimization and applications. It can be further divided into two subsidiary categories: multi-agent cooperation optimization, and clustering or classification optimization. For specific applications, they are formulated as the optimization problem and solved by novel computational intelligence algorithms.

Marco Severini, Stefano Squartini, Francesco Piazza address the problem of task scheduling in processors located in sensor nodes powered by energy harvesting sources. With the aim of achieving a more efficient and conservative management of energy resources, a new improved Lazy Scheduling Algorithm is proposed in order to reduce the conventional LSA computational complexity and maximize the amount of energy available for task execution. The proposed method can predict the occurrence of task energy starving, allows to obtain a more conservative and efficient management of energy with respect to the original LSA.

Quande Yuan and Yi Guan et al. investigate the multirobot task allocation problem using contract net protocol (CNP) with neural network. CNP is a negotiation model and can solve resources conflict through allocating tasks to robots using market mechanisms. In the method of CNP, a decision-making function is usually used. However, it is difficult to find a function to evaluate robots bids when each robot gives more than one bid price to reflect its different abilities. In order to overcome the difficulty of constructing a decision function to evaluate robot bidders' prices, an improved BP neural network is applied to fuse these prices and decide which robot is the successful bidder. The experiment result shows the effectiveness of the proposed method.

Hairong Dong et al. investigate the problem of controlling multiple mobile agents to maintain a desired geometric formation within a constrained environment. The effect of size and initial velocity of multiple mobile agents for the formation control has been considered by designing a fuzzy logic-based controller in a given constraint environment. By designing the fuzzy logic-based formation control law, the entire group moves into the given constrained environment as a desired shape. All agent velocities asymptotically approach the velocity value of the leader, and collisions are avoided between agents and the 
environment boundary. Numerical simulations demonstrate the effectiveness of the proposed controller.

The following 4 papers belong to the data-based optimization for clustering or classification sub-category.

Classification problem of multiple observation sets via network modularity is studied by Thiago H. Cupertino, Thiago C. Silva, and Liang Zhao. A new network-based method for the classification of multiple observation sets is proposed, which uses a network representation of the input data to take advantage of the topological relations between the patterns revealed by a low-dimensional manifold. A measurement called modularity is computed to numerically indicate the topological characteristics of the constructed networks. The simulation results show that the proposed method performed well in a handwritten digits database and a multiple view object collections.

Motor imagery classification problem is studied by Sanqing $\mathrm{Hu}, \mathrm{Yu} \mathrm{Cao}$, and Qiangqiang Tian by applying joint regression model and spectral power of EEG data for classification during motor imagery states. Firstly, time domain feature and frequency domain feature in particular bands are introduced, and then two features are combined together as a vector for which many classifiers can be used to classify. The EEG data sets are obtained from the $\mathrm{Cp} 3 /$ Cp4 or C3/C4 electrode locations of the standard 10/20 system. The algorithm produces a classification accuracy of $90 \%$ on the training data of one subject from BCI2003 data set III and $80 \%$ on the test data. The results are better than that by using auto-regression model.

Part-based models are getting popular for human detection in still images due to its flexibility and robustness in modeling relative positions of object parts, but most researches assume that the training data is well aligned and the detector should get highest response at the annotated area. It is difficult to tackle misalignment of samples, which is often the case in real applications. Jefferson Shen, Wankou Yang, and Changyin Sun present a variable granularity HOG-CSLBP feature, which combines the human shape information with local texture information and encodes spatial relationship in different granularity. The new feature takes advantage of the mutual complementarities of histogram of gradient (HOG) and centersymmetric local binary patterns (CSLBP) feature. Then, a Gentle MILBoost algorithm is presented, which utilizes the Newton update technique to get an optimal weak classifier. This approach is demonstrated to be efficient and numerically stable.

Circulating fluidized bed boiler is a complicated control object, and traditional control methods cannot obtain good control effect, which becomes one of the bottlenecks for its extending application. Niu Peifeng and Ma Yunfei et al. propose a cluster control method based on the analyses of dynamic characteristics in circulating fluidized bed boiler burning control. This method is on the basis of information fusion technology using hybrid neural network algorithm. The proposed method can fuse and classify the data sampled by all the sensors and achieve the corresponding control by judging corresponding policy space of different data.

The papers in the last category lie in the area of databased modeling, with the use of input and output data, by means of several kinds of computational intelligence methods, aiming at different application issues. No subsidiary categories are provided here; however, the papers are indexed as the computational intelligence methods: neural networks, fuzzy logic systems, and evolutionary computation algorithms.

Multistability problem of periodic delayed recurrent neural network with memristors is discussed by Gang Bao and Zhigang Zeng. According to this property of memristor, the coefficients of RNN with memristors are periodic functions with respect to time, and this kind of model can be used to describe evolutionary processes of the biological systems under a periodically varying environment. By dividing the state space and using contraction mapping theorem, one sufficient condition is obtained for multiperiodicity and the periodic orbits located in saturation regions are locally exponentially stable limit cycles.

Radial basis function (RBF) neural networks have been widely used in various applications. Unlike parameter optimization, determining an appropriate structure for an RBF neural model is still an open problem. Zhou Quan, Song Shiji, and Wu Cheng present an effective method to construct compact RBF neural networks, named kernelized LARS-LASSO. The LARS is efficient for computing the whole L1 regularization path, while the LASSO naturally links the L1-constrained models to the regressor terms selection and weights shrinkage. The proposed algorithm simultaneously conducts model structure and parameter optimization, which leads to better generalization performance of the network than previous approaches.

Hong-Jie Xing and Xin-Mei Wang propose a regularized correntropy criterion (RCC) for extreme learning machine (ELM) to deal with the training set with noises or outliers, in which Gaussian kernel function is utilized to substitute Euclidean norm of the mean square error criterion. The proposed method makes ELM-RCC more robust against noises than that of ELM. In order to further improve the generalization ability of ELM-RCC, a regularization term is added into its objective function. Meanwhile, the related propositions of the proposed method, the algorithmic implementation, and computational complexity of ELM-RCC are also provided. Moreover, the optimal weights connecting the hidden and output layers together with the optimal bias terms can be promptly obtained by the half-quadratic optimization technique with an iterative manner. 
Type-2 fuzzy logic can deal with high level uncertainties that cannot be handled by classic fuzzy logic (type-1 fuzzy logic). When combined with neural network, termed T2FNN, its ability can be greatly enhanced in modeling complex systems. However, in many systems, exact physical structure knowledge is lacked, and what we only know is some kind of qualitative knowledge. One particular but common qualitative knowledge is the monotonicity property between the inputs and outputs. Chengdong Li, Jianqiang Yi et al. derive some sufficient conditions on the parameters of T2FNNs to ensure the monotonicity between the inputs and outputs. An algorithm is then proposed to optimize the parameters of the T2FNNs and validated on the thermal comfort index prediction. Experiments demonstrate the advantage of the monotonic T2FNN over other methods.

Gathering holistic sensor measurements in wireless sensor network is often difficult due to stringent constraint on network resources, frequent link, indeterminate variations in sensor readings, and node failures. Xin Song, Cuirong Wang et al. present a distributed linear regression-based data gathering framework in clustered wireless sensor networks. Cluster head nodes in the network maintain linear regression model and use historical sensory data to perform estimation of the actual monitoring measurements. They communicate the constraints on the model parameters with the sink nodes. The proposed framework can implement sensory data prediction and extraction with tolerable error bound. In addition, it can achieve more energy savings than other schemes.

Chao Zhou, Zeng-Guang Hou et al. focus on an interesting problem: How to make a robotic fish swim backward.
In many circumstances, for example, when the robot works in confined gaps, pipelines, and maze where the space is too narrow for it to turn back, swimming backward is important. Most existing researches concern about symmetric robotic fish between the head and tail, and backward swimming can be realized easily by exchanging the motion law of anterior links with the posterior ones. But in this work, the authors study a carangiform robotic fish which has an asymmetric body. An effective method for searching gaits during backward swimming is proposed by mimicking the European eel's freely swimming mode.

A fault diagnosis method of smart grid based on rough set combined with genetic algorithm and Tabu search is proposed by Qiuye Sun and Chunling Wang et al., in order to improve the accuracy, robustness, and speed of the fault location. The reductions in continuous attributes and value are the major application of rough sets. The proposed algorithm can combine the parallel global searching capability of genetic algorithm with the local searching ability of Tabu search and significantly improve the algorithm efficiency of execution and ensure global optimal results. The effectiveness of the proposed algorithm has been demonstrated by three kinds of fault classification tests in Changchun south substation and its distribution grid.

The above briefly introduces 20 papers in this special issue. Thanks again for authors to share their valuable work with us. At last but not least, we would be very grateful to the Neural Computation and Applications editorial staff for their patience and precious support! 